\title{
Bifurcation Analysis and Control of a Differential-Algebraic Predator-Prey Model with Allee Effect and Time Delay
}

\author{
Xue Zhang and Qing-ling Zhang \\ Department of Science, Northeastern University, No. 3-11, Wenhua Road, Heping District, Shenyang, Liaoning 110004, China \\ Correspondence should be addressed to Xue Zhang; zhangxueer@gmail.com
}

Received 3 August 2013; Accepted 22 January 2014; Published 3 March 2014

Academic Editor: Junjie Wei

Copyright ( 2014 X. Zhang and Q.-1. Zhang. This is an open access article distributed under the Creative Commons Attribution License, which permits unrestricted use, distribution, and reproduction in any medium, provided the original work is properly cited.

\begin{abstract}
This paper studies systematically a differential-algebraic prey-predator model with time delay and Allee effect. It shows that transcritical bifurcation appears when a variation of predator handling time is taken into account. This model also exhibits singular induced bifurcation as the economic revenue increases through zero, which causes impulsive phenomenon. It can be noted that the impulsive phenomenon can be much weaker by strengthening Allee effect in numerical simulation. On the other hand, at a critical value of time delay, the model undergoes a Hopf bifurcation; that is, the increase of time delay destabilizes the model and bifurcates into small amplitude periodic solution. Moreover, a state delayed feedback control method, which can be implemented by adjusting the harvesting effort for biological populations, is proposed to drive the differential-algebraic system to a steady state. Finally, by using Matlab software, numerical simulations illustrate the effectiveness of the results.
\end{abstract}

\section{Introduction}

In recent years, the growing human needs for more food and more energy have led to increased exploitation of these resources. The problems related to many fields like fishery, forestry, and wildlife. Therefore, mankind is facing the dual problems of resource shortages and environmental degradation. Concerning the conservation for the long-term benefits of humanity, there is a wide-range of interest in analysis and modelling of bioeconomic systems. In many earlier studies, it has been shown that harvesting has a strong impact on population dynamics, ranging from rapid depletion to complete preservation of biological populations. Two main kinds of harvesting were focused on nonzero constant harvesting [1-3] and constant harvesting effort [4-7]. With constant harvesting, the generalized Gause prey-predator model is found to exhibit saddle-node bifurcations, Hopf bifurcation, heteroclinic bifurcation, and nilpotent saddle bifurcation [1]. Xiao et al. [2] have investigated the dynamical properties of a ratio-dependent predator-prey model with nonzero constant rate predator harvesting. These results reveal far richer dynamics compared to the model with no harvesting.
The literature [3] shows that harvesting effort as control parameter is not only possible to control the cyclic behavior of populations leading to the persistence of all species, but other desired stable equilibrium including disease-free can also be obtained. Das et al. [4] discussed the bioeconomic harvesting of a prey-predator fishery in which both species are infected by some toxicant. Ji and $\mathrm{Wu}$ [5] studied a predator-prey model with a constant-rate prey harvesting incorporating a constant prey refuge, where the influence of harvesting effort on the density of two species was discussed. Chakraborty et al. [6] describes a prey-predator model with stage structure for predator and selective harvesting effort on predator population. Geometric approach is used to drive the sufficient conditions for global stability of the system, and fishing effort is used to investigate the optimal utilization of the resource. Xiang et al. [7] consider a Lotka-Volterra model with impulsive harvest for the prey and investigate globally attractive periodic solution. However, most of these discussions are only based on differential equations or difference equations.

In power systems, neural networks, and genetic networks [8-12], differential-algebraic equations have been studied 
widely and a lot of results have been obtained, such as local stability, optimal control, singularity induced bifurcation, and feasibility regions. From 2009, several differentialalgebraic biological models were reported [13-16]. Surprisingly enough, all the existing differential-algebraic biological modelling literature considers only the simplest case of a logistic prey growth function. However, numerous examples demonstrate that the growth of natural populations can exhibit Allee effect, which is a phenomenon in biology named after Allee [17]. Allee effect describes a positive relation between population density and the per capita growth rate. In other words, for smaller populations, the reproduction and survival of individuals decrease. This effect usually saturates or disappears as populations get larger. The effect may be due to any number of causes, for example, mate finding, social dysfunction, inbreeding depression, food exploitation, and predator avoidance or defense.

On the other hand, since reproduction of predator after consuming prey is not instantaneous in most cases, some time lag for gestation is required. Therefore, in this paper, we consider a differential-algebraic prey-predator model with time delay and the Allee effect on the growth of the prey population. We analyze the stability properties and bifurcation behavior of this model. A state delayed feedback control method is also proposed, which can eliminate Hopf bifurcation and drive the differential-algebraic prey-predator model to stay at a steady state.

\section{Model Equations}

The general predator-prey model in its classical form is represented by

$$
\frac{d \tilde{x}}{d \tilde{t}}=\tilde{x} p(\tilde{x})-\tilde{y} q(\tilde{x}), \quad \frac{d \tilde{y}}{d \tilde{t}}=\beta \tilde{y} q(\tilde{x})-d_{2} \tilde{y},
$$

where $\tilde{x}$ and $\tilde{y}$ represent the prey density and predator density at time $\tilde{t}$, respectively; $p(\tilde{x})$ is the per capita growth rate of prey in absence of predator; $d_{2}$ is the intrinsic mortality rate of predator in the absence of food; $q(\tilde{x})$ is predator's functional response, defined as the amount of prey catch per predator per unit of time; $\beta(0<\beta<1)$ is the rate of conversion of nutrients into the reproduction rate of the predator.

In 1954, Gordon [18] studied the effect of harvest effort on ecosystem from an economic perspective and proposed the following economic theory:

\section{Net Economic Revenue (NER)}

$$
=\text { Total Revenue }(\mathrm{TR})-\text { Total Cost }(\mathrm{TC}) \text {. }
$$

Based on this theory, this paper studies a class of delayed differential-algebraic predator-prey model with Allee effect on prey species and Holling-II functional response, which is written in the following form:

$$
\begin{gathered}
\frac{d \tilde{x}}{d \tilde{t}}=\tilde{x}\left(s(\tilde{x})-m_{1}-a \tilde{x}(\tilde{t}-\tilde{\tau})\right)-\frac{\alpha \tilde{x} \tilde{y}}{1+\alpha \tilde{h} \tilde{x}}, \\
\frac{d \tilde{y}}{d \tilde{t}}=\frac{\beta \alpha \tilde{x}(\tilde{t}-\tilde{\tau}) \tilde{y}}{1+\alpha \tilde{h} \tilde{x}(\tilde{t}-\tilde{\tau})}-m_{2} \tilde{y}-\widetilde{E} \tilde{y} \\
0=\tilde{E}(p \tilde{y}-c)-m,
\end{gathered}
$$

where $s(\tilde{x})$ is the fertility rate of prey species. $m_{1}$ and $m_{2}$ are the intrinsic mortality rate of the prey and predator species, respectively. $a$ is the strength of intracompetition of prey population. $\alpha$ is the attack coefficient and $\tilde{h}$ is the handling time. $\beta$ denotes food utilization efficiency. The predator takes time $\widetilde{\tau}$ to convert the food into its growth; $\widetilde{E}$ is harvesting effort for predator, $p>0, c>0$, and $m>0$ are harvesting reward per unit harvesting effort for unit weight of predator, harvesting cost per unit harvesting effort for predator, and the net economic revenue per unit harvesting effort, respectively. All the parameters are positive constants.

Let the fertility rate $s(\tilde{x})$ increase with population density and be described by

$$
s(\tilde{x})=\frac{r \tilde{x}}{\widetilde{A}+\tilde{x}},
$$

where $r>0$ and $\widetilde{A}$ are the per capita maximum fertility rate and the Allee effect constant of the prey species, respectively. If $\widetilde{A}>0$, the fertility of the species is zero when $\tilde{x}$ is zero and approaches to $r$ when $\tilde{x}$ becomes very large. The increasing of $s(\widetilde{x})$ depends on the parameter $\widetilde{A}$. The larger $\widetilde{A}$ is, the stronger Allee effect will be. In particular, the fertility rate is density independent when $\widetilde{A}=0$; that is, $s(\widetilde{x})=r$.

When the prey population is subject to Allee effect above, the predator-prey model (3) becomes

$$
\begin{gathered}
\frac{d \tilde{x}}{d \tilde{t}}=\tilde{x}\left(\frac{r \tilde{x}}{\widetilde{A}+\tilde{x}}-m_{1}-a \tilde{x}(\tilde{t}-\tilde{\tau})\right)-\frac{\alpha \tilde{x} \tilde{y}}{1+\alpha \tilde{h} \tilde{x}}, \\
\frac{d \tilde{y}}{d \tilde{t}}=\frac{\beta \alpha \tilde{x}(\tilde{t}-\tilde{\tau}) \tilde{y}}{1+\alpha \tilde{h} \tilde{x}(\tilde{t}-\tilde{\tau})}-m_{2} \tilde{y}-\tilde{E} \tilde{y} \\
0=\tilde{E}(p \tilde{y}-c)-m .
\end{gathered}
$$

Assume that the per capita maximum fertility rate of prey must exceed its death rate, that is, $r>m_{1}$; otherwise, both prey and predator will become extinct. In addition, the maximum growth rate of predator population must exceed its death rate, that is, $\beta / \widetilde{h}>m_{2}$. If not, prey population will never be able to sustain predator population.

\section{Qualitative Analysis}

We nondimensionalize the model (5) with the following scaling:

$$
x=\frac{a \tilde{x}}{r}, \quad y=\frac{\alpha \tilde{y}}{r}, \quad t=r \tilde{t},
$$


and then obtain the following form:

$$
\begin{gathered}
\frac{d x}{d t}=x\left(\frac{x}{A+x}-d_{1}-x(t-\tau)\right)-\frac{x y}{1+h x}, \\
\frac{d y}{d t}=y\left(\frac{b x(t-\tau)}{1+h x(t-\tau)}-d_{2}\right)-E y, \\
0=E(v y-w)-m,
\end{gathered}
$$

where the nondimensional parameters are defined as

$$
\begin{gathered}
A=\frac{a \widetilde{A}}{r}, \quad d_{1}=\frac{m_{1}}{r}, \quad h=\frac{\alpha r \tilde{h}}{a}, \quad b=\frac{\alpha \beta}{a}, \\
d_{2}=\frac{m_{2}}{r}, \quad E=\frac{\widetilde{E}}{r}, \quad v=\frac{p r^{2}}{\alpha}, \quad w=r c, \\
\tau=r \tilde{\tau} .
\end{gathered}
$$

From the assumption mentioned above, the following reasonable condition must be satisfied:

$$
0<d_{1}<1
$$

For simplicity of computation, we consider the above model (7) instead of the model (5). Hence, we will perform a qualitative analysis of the model (7).

Let

$$
\begin{aligned}
f(X, E, \mu)= & \left(\begin{array}{l}
f_{1}(X, E, \mu) \\
f_{2}(X, E, \mu)
\end{array}\right) \\
= & \left(\begin{array}{c}
x\left(\frac{x}{A+x}-d_{1}-x(t-\tau)\right)-\frac{x y}{1+h x} \\
y\left(\frac{b x(t-\tau)}{1+h x(t-\tau)}-d_{2}\right)-E y
\end{array}\right), \\
& g(X, E, \mu)=E(v y-w)-m=0,
\end{aligned}
$$

where $X=(x, y)^{T}$ is the state and $\mu$ denotes bifurcation parameter.
3.1. The Model (7) with Zero Economic Profit. Considering zero economic revenue, the model (7) can be reduced as follows:

$$
\begin{gathered}
\frac{d x}{d t}=x\left(\frac{x}{A+x}-d_{1}-x(t-\tau)\right)-\frac{x y}{1+h x} \\
\frac{d y}{d t}=y\left(\frac{b x(t-\tau)}{1+h x(t-\tau)}-d_{2}\right)-E y, \\
0=E(v y-w) .
\end{gathered}
$$

By the analysis of roots for the model (11), we obtain the following result.

Theorem 1. (1) The model (11) has a trivial equilibrium point $Q_{0}(0,0,0)$ for any positive parameters.

(2) There exist two boundary equilibrium points $Q_{1}\left(x_{1}, 0,0\right)$ and $Q_{2}\left(x_{2}, 0,0\right)$ if $1-A-d_{1}>2 \sqrt{A d_{1}}$, where

$$
\begin{aligned}
& x_{1}=\frac{1}{2}\left(1-A-d_{1}-\sqrt{\left(1-A-d_{1}\right)^{2}-4 A d_{1}}\right), \\
& x_{2}=\frac{1}{2}\left(1-A-d_{1}+\sqrt{\left(1-A-d_{1}\right)^{2}-4 A d_{1}}\right) .
\end{aligned}
$$

(3) There exists another boundary equilibrium point $Q_{3}\left(x_{3}, y_{3}, 0\right)$, where $x_{3}=d_{2} / \delta, y_{3}=b\left[\delta d_{2}-\left(d_{1} \delta+d_{2}\right)(A \delta+\right.$ $\left.\left.d_{2}\right)\right] / \delta^{2}\left(A \delta+d_{2}\right), \delta=b-d_{2} h$, if the following conditions are satisfied:

$$
\begin{gathered}
0<d_{1}<1, \quad b>\frac{d_{2}\left(1+\left(1-d_{1}\right) h\right)}{1-d_{1}}, \\
0<A<\frac{d_{2}\left(\delta\left(1-d_{1}\right)-d_{2}\right)}{\delta\left(d_{2}+d_{1} \delta\right)} .
\end{gathered}
$$

(4) The model (11) has a positive equilibrium point $Q_{4}\left(x_{4}, y_{4}, E_{4}\right)$, where $y_{4}=w / v, E_{4}=\left(b x_{4}\right) /\left(1+h x_{4}\right)-d_{2}$, and $x_{4}$ is the root of the following equation:

$$
\begin{aligned}
v h x^{3} & +v\left(h\left(d_{1}+A-1\right)+1\right) x^{2} \\
& +\left(v\left(d_{1} A h+d_{1}+A-1\right)+w\right) x \\
& +d_{1} A v+w A=0 .
\end{aligned}
$$
form:

The Jacobian matrix of the model (11) takes the following

$$
J=D_{X} f-D_{E} f\left(D_{E} g\right)^{-1} D_{X} g=\left(\begin{array}{cc}
\frac{x(x+2 A)}{(A+x)^{2}}-d_{1}-x-x e^{-\lambda \tau}-\frac{y}{(1+h x)^{2}} & -\frac{x}{1+h x} \\
\frac{b y e^{-\lambda \tau}}{(1+h x)^{2}} & \frac{b x}{1+h x}-d_{2}-E+\frac{v E y}{v y-w}
\end{array}\right)
$$

Hence, the Jacobian matrix of the model (11) at $Q_{0}$ is

$$
J_{\mathrm{Q}_{0}}=\left(\begin{array}{cc}
-d_{1} & 0 \\
0 & -d_{2}
\end{array}\right)
$$

Theorem 2. $Q_{0}$ is always a locally stable node.

From Theorem 2, it can be seen that both prey and predator populations will become extinct when their population densities lie in the attraction region of $Q_{0}$. 
Note that $x_{1} /\left(A+x_{1}\right)-d_{1}-x_{1}=0$, then the Jacobian matrix of the model (11) at $Q_{1}$ is

$$
\begin{aligned}
& J_{\mathrm{Q}_{1}} \\
& =\left(\begin{array}{cc}
\frac{x_{1}}{A+x_{1}}\left(1-d_{1}-x_{1}-\left(A+x_{1}\right) e^{-\lambda \tau}\right) & -\frac{x_{1}}{1+h x_{1}} \\
0 & \frac{b x_{1}}{1+h x_{1}}-d_{2}
\end{array}\right) .
\end{aligned}
$$

Since

$$
\begin{aligned}
1-d_{1}-x_{1}-\left(A+x_{1}\right) e^{-\lambda \tau} & >1-d_{1}-2 x_{1}-A \\
& =\sqrt{\left(1-A-d_{1}\right)^{2}-4 A d_{1}}>0
\end{aligned}
$$

and the other eigenvalue of $J_{\mathrm{Q}_{1}}$ is $b x_{1} /\left(1+h x_{1}\right)-d_{2}$, we obtain the following results on the stability of $Q_{1}$.

Theorem 3. Assume that $0<d_{1}<1$ and $0<A<\left(1-\sqrt{d_{1}}\right)^{2}$. Then, for any time delay $\tau \geq 0$, one has the following.

(1) $Q_{1}$ is a saddle point if

$$
h>\frac{b}{d_{2}}-\frac{2}{1-A-d_{1}-\sqrt{\left(1-A-d_{1}\right)^{2}-4 A d_{1}}} .
$$

(2) $Q_{1}$ is an unstable node if

$$
0<h<\frac{b}{d_{2}}-\frac{2}{1-A-d_{1}-\sqrt{\left(1-A-d_{1}\right)^{2}-4 A d_{1}}} .
$$

Next, we analyze the stability of the equilibrium point $Q_{2}$. The Jacobian matrix of the model (11) at $Q_{2}$ is

$J_{Q_{2}}$

$$
=\left(\begin{array}{cc}
\frac{x_{2}}{A+x_{2}}\left(1-d_{1}-x_{2}-\left(A+x_{2}\right) e^{-\lambda \tau}\right) & -\frac{x_{2}}{1+h x_{2}} \\
0 & \frac{b x_{2}}{1+h x_{2}}-d_{2}
\end{array}\right) .
$$

For $Q_{2}$, one eigenvalue is $b x_{2} /\left(1+h x_{2}\right)-d_{2}$, and the other is given by the equation

$$
\lambda-\frac{x_{2}}{A+x_{2}}\left(1-d_{1}-x_{2}-\left(A+x_{2}\right) e^{-\lambda \tau}\right)=0 .
$$

By simple analysis, the root of (22) is negative if the time delay satisfies $\tau<\tau_{0}$, where

$$
\begin{aligned}
\tau_{0}= & \frac{A+x_{2}}{x_{2} \sqrt{\left(1-d_{1}+A\right)\left(A-1+d_{1}+2 x_{2}\right)}} \\
& \times \arccos \frac{1-d_{1}-x_{2}}{A+x_{2}} .
\end{aligned}
$$

Then, we have the following results on the stability of $Q_{2}$.
Theorem 4. Assume that $0<d_{1}<1,0<A<\left(1-\sqrt{d_{1}}\right)^{2}$, and $\tau<\tau_{0}$. Then, one has the following.

(1) $Q_{2}$ is a saddle point if

$$
0<h<\frac{b}{d_{2}}-\frac{2}{1-A-d_{1}+\sqrt{\left(1-A-d_{1}\right)^{2}-4 A d_{1}}} .
$$

(2) $Q_{2}$ is locally asymptotically stable if

$$
h>\frac{b}{d_{2}}-\frac{2}{1-A-d_{1}+\sqrt{\left(1-A-d_{1}\right)^{2}-4 A d_{1}}} .
$$

Based on Theorem 4, the following bifurcation result can be obtained.

Theorem 5. Assume that $0<d_{1}<1,0<A<\left(1-\sqrt{d_{1}}\right)^{2}$, and $\tau<\tau_{0}$. Then, the model (11) undergoes transcritical bifurcation at the equilibrium point $Q_{2}$ when bifurcation parameter $h$ increases through $h_{0}=b / d_{2}-2 /\left(1-A-d_{1}+\right.$ $\sqrt{\left.\left(1-A-d_{1}\right)^{2}-4 A d_{1}\right)}$.

Proof. When $\tau<\tau_{0}$ and the bifurcation parameter $h=h_{0}$, the characteristic polynomial at the equilibrium point $Q_{2}$ has a simple zero eigenvalue with left null vector

$$
u_{\text {trans }}=\left(\begin{array}{ll}
0 & 1
\end{array}\right)
$$

and right null vector

$$
\begin{aligned}
& v_{\text {trans }}=\left(\frac{x_{2}}{1+h x_{2}} \frac{x_{2}}{A+x_{2}}\left(1-d_{1}-2 x_{2}-A\right)\right)^{T}, \\
& \left.u_{\text {trans }}\left(D_{X} D_{h} f_{R}\right) v_{\text {trans }}\right|_{Q_{2}} \\
& =\left(\begin{array}{ll}
0 & 1
\end{array}\right)\left(\begin{array}{cc}
\frac{2 x_{2} y_{2}}{\left(1+h x_{2}\right)^{3}} & \frac{x_{2}^{2}}{\left(1+h x_{2}\right)^{2}} \\
-\frac{2 b x_{2} y_{2}}{\left(1+h x_{2}\right)^{3}} & -\frac{b x_{2}^{2}}{\left(1+h x_{2}\right)^{2}}
\end{array}\right) \\
& \times\left(\begin{array}{c}
\frac{x_{2}}{1+h x_{2}} \\
\frac{x_{2}\left(1-d_{1}-2 x_{2}-A\right)}{A+x_{2}}
\end{array}\right) \\
& =-\frac{b x_{2}^{3}\left(1-d_{1}-2 x_{2}-A\right)}{\left(1+h x_{2}\right)^{2}\left(A+x_{2}\right)} \text {, }
\end{aligned}
$$

$$
\begin{aligned}
\left.u_{\text {trans }} D_{X}^{2} f_{R}\left(v_{\text {trans }}, v_{\text {trans }}\right)\right|_{P_{2}^{0}} & =\left(\begin{array}{ll}
0 & 1
\end{array}\right)\left(\begin{array}{c}
-\frac{x_{2}}{\left(1+h x_{2}\right)^{3}} \\
\frac{b x_{2}}{\left(1+h x_{2}\right)^{3}}
\end{array}\right) \\
& =\frac{b x_{2}}{\left(1+h x_{2}\right)^{3}} .
\end{aligned}
$$

According to the literature [19], the model (11) undergoes transcritical bifurcation at the equilibrium point $Q_{2}$. This completes the proof. 
Remark 6. Transcritical bifurcation implies that the equilibrium point $Q_{2}$ remains stable if the handling time is longer than the critical point $h_{0}$, otherwise the stability of $Q_{2}$ is lost. From the view of biological explanation, stable equilibrium point $Q_{2}$ means that predator population is to be extinct, which results from longer handling time reducing the amount of prey catch per predator per unit of time.

The Jacobian matrix of the model (11) at $Q_{3}$ is

$$
J_{Q_{3}}=\left(\begin{array}{cc}
\frac{x_{3}\left(x_{3}+2 A\right)}{\left(A+x_{3}\right)^{2}}-d_{1}-x_{3}-\frac{y_{3}}{\left(1+h x_{3}\right)^{2}}-x_{3} e^{-\lambda \tau} & -\frac{x_{3}}{1+h x_{3}} \\
\frac{b y_{3} e^{-\lambda \tau}}{\left(1+h x_{3}\right)^{2}} & 0
\end{array}\right)
$$

The corresponding characteristic equation is

$$
\begin{aligned}
\lambda^{2}- & \left(\frac{A x_{3}}{\left(A+x_{3}\right)^{2}}+\frac{h x_{3} y_{3}}{\left(1+h x_{3}\right)^{2}}\right) \lambda \\
& +\left(x_{3} \lambda+\frac{b x_{3} y_{3}}{\left(1+h x_{3}\right)^{3}}\right) e^{-\lambda \tau}=0 .
\end{aligned}
$$

In the absence of delay, since the constant term of the above equation is always positive, the sign of the following expression:

$$
x_{3}-\frac{A x_{3}}{\left(A+x_{3}\right)^{2}}-\frac{h x_{3} y_{3}}{\left(1+h x_{3}\right)^{2}}
$$

determines the stability of equilibrium $Q_{3}$. Moveover, if the following condition:

$$
\delta\left(4 b d_{1} d_{2} h-\left(b+d_{2} h\right)^{2}\right)+4 b d_{2}\left(b+d_{2} h\right)>0
$$

is satisfied, it is clear that $Q_{3}$ is always locally asymptotically stable for the model (11) in the absence of delay.

In the presence of delay, assume that a purely imaginary solution of the form $\lambda=i \omega_{0}$ exists for the above characteristic equation, where $\omega_{0}$ is the root of the following equation:

$$
\begin{gathered}
\omega^{4}+\left[\left(\frac{A x_{3}}{\left(A+x_{3}\right)^{2}}+\frac{h x_{3} y_{3}}{\left(1+h x_{3}\right)^{2}}\right)^{2}-x_{3}^{2}\right] \omega^{2} \\
-\frac{b^{2} x_{3}^{2} y_{3}^{2}}{\left(1+h x_{3}\right)^{6}}=0 .
\end{gathered}
$$

Obviously, this equation has a positive solution $\omega_{0}$. Therefore, the system undergoes Hopf bifurcation at the equilibrium point $Q_{3}$ when

$$
\begin{aligned}
\tau_{k}^{0} & \\
=\frac{1}{\omega_{0}} \arccos ( & \left.\left.+\frac{h x_{3} y_{3}}{\left(1+h x_{3}\right)^{2}}\right)\right) \\
& \times\left(\frac{b y_{3}}{\left(1+h x_{3}\right)^{3}}+\frac{A x_{3}}{\left(A+x_{3}\right)^{2}}\right. \\
& \left.\left(x_{3} \omega_{0}^{2}+\frac{b^{2} x_{3} y_{3}^{2}}{\left(1+h x_{3}\right)^{6}}\right)^{-1}\right)+\frac{2 k \pi}{\omega_{0}} .
\end{aligned}
$$

Then the following result is obtained.

Theorem 7. Assume that the condition (31) holds. The model (11) is stable for the time delay $\tau<\tau_{0}^{0}$ and undergoes Hopf bifurcation at the equilibrium point $Q_{3}$ when $\tau=\tau_{0}^{0}$.

In succession, we discuss the bifurcation behavior regarding $m$ as the bifurcation parameter; that is, $\mu=m$.

Theorem 8. If $A /\left(A+x_{4}\right)^{2}+h y_{4} /\left(1+h x_{4}\right)^{2} \neq 1$, the model (11) undergoes singular induced bifurcation at the equilibrium point $Q_{4}$ when the bifurcation parameter $\mu$ increases through 0 . Moreover, the stability of the equilibrium point $Q_{4}$ changes, that is, from stable to unstable.

Proof. We define a new variable as $\Delta=D_{E} g=v y-w$. Then, $\Delta$ has a simple zero eigenvalue at the equilibrium point $Q_{4}\left(x_{4}, y_{4}, E_{4}\right)$ as follows:

$$
\begin{aligned}
& \left|\begin{array}{cc}
D_{X} f & D_{E} f \\
D_{X} g & D_{E} g
\end{array}\right|_{Q_{4}} \\
& \quad=\left|\begin{array}{ccc}
\frac{A x_{4}}{\left(A+x_{4}\right)^{2}}-x_{4}+\frac{h x_{4} y_{4}}{\left(1+h x_{4}\right)^{2}} & -\frac{x_{4}}{1+h x_{4}} & 0 \\
\frac{b y_{4}}{\left(1+h x_{4}\right)^{2}} & 0 & -y_{4} \\
0 & v E_{4} & v y_{4}-w
\end{array}\right|
\end{aligned}
$$




$$
\begin{aligned}
& =v x_{4} y_{4} E_{4}\left(\frac{A}{\left(A+x_{4}\right)^{2}}-1+\frac{h y_{4}}{\left(1+h x_{4}\right)^{2}}\right) \text {, } \\
& \left.\operatorname{trace}\left(D_{E} f \operatorname{adj}\left(D_{E} g\right) D_{X} g\right)\right|_{Q_{4}}=\operatorname{trace}\left(\left(\begin{array}{c}
0 \\
-y_{4}
\end{array}\right)\left(\begin{array}{ll}
0 & v E_{4}
\end{array}\right)\right) \\
& =-w E_{4}, \\
& \left|\begin{array}{lll}
D_{X} & D_{E} f & D_{m} f
\end{array}\right| \\
& \begin{array}{lll}
D_{X} & D_{E} \mathcal{g} & D_{m} g
\end{array} \\
& \left.D_{X} \Delta D_{E} \Delta D_{m} \Delta\right|_{Q_{4}} \\
& =\left|\begin{array}{cccc}
\frac{A x_{4}}{\left(A+x_{4}\right)^{2}}-x_{4}+\frac{h x_{4} y_{4}}{\left(1+h x_{4}\right)^{2}} & -\frac{x_{4}}{1+h x_{4}} & 0 & 0 \\
\frac{b y_{4}}{\left(1+h x_{4}\right)^{2}} & 0 & -y_{4} & 0 \\
0 & v E_{4} & v p_{4}-w & -1 \\
0 & v & 0 & 0
\end{array}\right| \\
& =v x_{4} y_{4}\left(\frac{A}{\left(A+x_{4}\right)^{2}}-1+\frac{h y_{4}}{\left(1+h x_{4}\right)^{2}}\right) \text {. }
\end{aligned}
$$

On the other hand, we get

$$
\begin{aligned}
& \xi_{1}=-\left.\operatorname{trace}\left(D_{E} f \operatorname{adj}\left(D_{E} g\right) D_{X} g\right)\right|_{Q_{4}}=w E_{4}>0,
\end{aligned}
$$

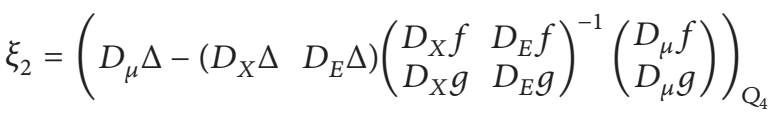

$$
\begin{aligned}
& =\frac{1}{E_{4}}>0 .
\end{aligned}
$$

According to [20], all conditions of singular induced bifurcation are satisfied. Hence, the model (11) undergoes singular induced bifurcation at the equilibrium point $Q_{4}$ if the economic revenue is zero. When economic revenue $m$ increases through 0 , one eigenvalue of the model (11) moves from $C^{-}$ (the open complex left half plane) to $C^{+}$(the open complex right half plane) along the real axis by diverging through $\infty$, which causes impulsive phenomenon of differential-algebraic system, that is, rapid expansion of the population from the view of biological explanation. Therefore, the stability of the equilibrium point $Q_{4}$ changes, that is, from stable to unstable. This completes the proof.

For the model (11) without time delay, eigenvalues of Jacobian matrix $J$ at the equilibrium point $Q_{4}$ are the roots of the following equation:

$$
\lambda^{2}-\left(a_{11}+a_{22}\right) \lambda+a_{11} a_{22}-a_{12} a_{21}=0,
$$

where

$$
\begin{gathered}
a_{11}=\frac{A x_{4}}{\left(A+x_{4}\right)^{2}}-x_{4}+\frac{h x_{4} y_{4}}{\left(1+h x_{4}\right)^{2}}, \\
a_{12}=-\frac{x_{4}}{1+h x_{4}}, \\
a_{21}=\frac{b y_{4}}{\left(1+h x_{4}\right)^{2}}, \quad a_{22}=\frac{v y_{4} E_{4}}{v y_{4}-w} .
\end{gathered}
$$

Remark 9. After simple computation, it can be seen that the Jacobian matrix $J$ at the equilibrium point $Q_{4}$ has two eigenvalues. One is

$$
\begin{aligned}
\lambda_{1}= & \frac{1}{2}\left[\left(a_{11}+a_{22}\right)+\sqrt{\left(a_{11}-a_{22}\right)^{2}+4 a_{12} a_{21}}\right] \\
& \longrightarrow+\infty\left(a_{11}\right),
\end{aligned}
$$

and the other is

$$
\begin{aligned}
\lambda_{2} & =\frac{1}{2}\left[\left(a_{11}+a_{22}\right)-\sqrt{\left(a_{11}-a_{22}\right)^{2}+4 a_{12} a_{21}}\right] \\
& \longrightarrow a_{11}(-\infty)
\end{aligned}
$$

since $a_{22} \rightarrow+\infty(-\infty)$ as $m \rightarrow 0^{+}\left(0^{-}\right)$.

3.2. The Model (7) with Positive Economic Profit. When the economic profit is positive, the model (7) has positive equilibrium point $Q_{5}\left(x_{5}, y_{5}, E_{5}\right)$, where coordinates $E_{5}=$ $m /\left(v y_{5}-w\right), y_{5}=\left(1+h x_{5}\right)\left(x_{5} /\left(A+x_{5}\right)-d_{1}-x_{5}\right)$, and $x_{5}$ are the root of the following equation:

$$
B(x)=B_{1} x^{4}+B_{2} x^{3}+B_{3} x^{2}+B_{4} x+B_{5}=0,
$$

where

$$
\begin{gathered}
B_{1}=v h\left(b-d_{2} h\right), \\
B_{2}=v\left(1+h\left(d_{1}+A-1\right)\right)\left(b-d_{2} h\right)-d_{2} v h, \\
B_{3}=\left(b-d_{2} h\right)\left(v h d_{1} A+\left(d_{1}+A-1\right) v+w\right) \\
-d_{2} v\left(1+h\left(d_{1}+A-1\right)\right)+m h, \\
B_{4}=\left(b-d_{2} h\right)\left(v d_{1}+w\right) A \\
-d_{2}\left(v h d_{1} A+\left(d_{1}+A-1\right) v+w\right) \\
+m(1+h A), \\
B_{5}=m A-d_{2} A\left(v d_{1}+w\right) .
\end{gathered}
$$

The Jacobian matrix of the model (7) at the equilibrium point $Q_{5}$ is

$$
J_{\mathrm{Q}_{5}}=\left(\begin{array}{cc}
\frac{A x_{5}}{\left(A+x_{5}\right)^{2}}-x_{5} e^{-\lambda \tau}+\frac{h x_{5} y_{5}}{\left(1+h x_{5}\right)^{2}} & -\frac{x_{5}}{1+h x_{5}} \\
\frac{b y_{5} e^{-\lambda \tau}}{\left(1+h x_{5}\right)^{2}} & \frac{v y_{5} E_{5}}{v y_{5}-w}
\end{array}\right) .
$$


For the sake of simplicity, let

$$
\begin{gathered}
u_{1}=u_{11}+u_{12}, \quad u_{11}=-x_{5}, \\
u_{12}=\frac{A x_{5}}{\left(A+x_{5}\right)^{2}}+\frac{h x_{5} y_{5}}{\left(1+h x_{5}\right)^{2}}, \\
u_{2}=-\frac{x_{5}}{1+h x_{5}}, \quad u_{3}=\frac{b y_{5}}{\left(1+h x_{5}\right)^{2}}, \\
u_{4}=\frac{v y_{5} E_{5}}{v y_{5}-w} .
\end{gathered}
$$

The characteristic polynomial for the model (7) without time delay at the equilibrium point $Q_{5}$ takes the following form:

$$
R_{1}(\lambda)=\lambda^{2}-\left(u_{1}+u_{4}\right) \lambda+u_{1} u_{4}-u_{2} u_{3}=0 .
$$

By using the Routh-Hurwitz criteria, $Q_{5}$ is locally asymptotically stable for the model (7) without time delay if

$$
u_{1}+u_{4}<0, \quad u_{1} u_{4}-u_{2} u_{3}>0
$$

According to the Jacobian matrix $J_{\mathrm{Q}_{5}}$, we can obtain the characteristic equation of the differential-algebraic model (7) at $Q_{5}$, which can be expressed as follows:

$$
R_{2}(\lambda)=\lambda^{2}+p_{1} \lambda+p_{2}+\left(p_{3} \lambda+p_{4}\right) e^{-\lambda \tau}=0
$$

where

$$
\begin{gathered}
p_{1}=-u_{12}-u_{4}, \quad p_{2}=u_{12} u_{4} \\
p_{3}=-u_{11}, \quad p_{4}=u_{11} u_{4}-u_{2} u_{3} .
\end{gathered}
$$

Assume that a purely imaginary solution of the form $\lambda=$ $i \omega$ exists in (46). Substituting it into (46) and separating the real and imaginary parts, we have

$$
\begin{gathered}
p_{2}-\omega^{2}+p_{4} \cos \omega \tau+p_{3} \omega \sin \omega \tau=0, \\
p_{1} \omega+p_{3} \omega \cos \omega \tau-p_{4} \sin \omega \tau=0 .
\end{gathered}
$$

Taking square on both sides of (48) and summing them up, we obtain

$$
\omega^{4}+\left(p_{1}^{2}-2 p_{2}-p_{3}^{2}\right) \omega^{2}+p_{2}^{2}-p_{4}^{2}=0 .
$$

From the condition (45), we have $p_{1}^{2}-2 p_{2}-p_{3}^{2}<0$ and $p_{2}+p_{4}>0$. Therefore, (49) has at least one real root $\omega_{m}$ if

$$
p_{2}-p_{4}<0
$$

The critical value of the delay corresponding to $\omega_{m}$ is given by

$$
\begin{array}{r}
\tau_{k}^{m}=\frac{1}{\omega_{m}} \arccos \frac{p_{4} \omega_{m}^{2}-p_{2} p_{4}-p_{1} p_{3} \omega_{m}^{2}}{p_{3}^{2} \omega_{m}^{2}+p_{4}^{2}}+\frac{2 k \pi}{\omega_{m}}, \\
k=0,1,2, \ldots .
\end{array}
$$

Now, differentiating (46) with respect to $\tau$, we obtain

$$
\frac{d \lambda}{d \tau}=\frac{\left(p_{3} \lambda+p_{4}\right) \lambda e^{-\lambda \tau}}{2 \lambda+p_{1}+\left[p_{3}-\tau\left(p_{3} \lambda+p_{4}\right)\right] e^{-\lambda \tau}} .
$$

Substituting the eigenvalue $i \omega_{m}$ and noticing that the derivative of (49) at $\omega_{m}^{2}$ is positive, it follows that

$$
\operatorname{sign}\left(\frac{d}{d \tau} \operatorname{Re}(\lambda)\right)_{\tau_{k}^{m}}=\operatorname{sign}\left(\omega_{m}^{2}\left(2 \omega_{m}^{2}+p_{1}^{2}-2 p_{2}-p_{3}^{2}\right)\right)>0
$$

Theorem 10. Assume that conditions (45) and (50) hold. For the model (7), there exists a $\tau_{0}^{m}>0$ such that $Q_{5}$ is locally asymptotically stable when $\tau \in\left[0, \tau_{0}^{m}\right)$ and is unstable when $\tau>\tau_{0}^{m}$. Furthermore, the model (7) undergoes Hopf bifurcation at the equilibrium point $Q_{5}$ when $\tau=\tau_{k}^{m}, k=$ $0,1,2, \ldots$..

From Theorem 10, it can be seen that Hopf bifurcation results in oscillation of population density; that is, the differential-algebraic model (7) becomes unstable when the time delay exceeds the critical value. From the view of ecological managers, it may be desirable to have stable population densities in order to keep sustainable development of ecosystem. However, food conversion delay is generally regarded as an inherence of biological populations. It is difficult to adjust the food conversion delay. Therefore, we propose the following state delayed feedback control method:

$$
\begin{gathered}
\frac{d x}{d t}=x\left(\frac{x}{A+x}-d_{1}-x(t-\tau)\right)-\frac{x y}{1+h x} \\
+L_{1}(x-x(t-\tau)), \\
\frac{d y}{d t}=y\left(\frac{b x(t-\tau)}{1+h x(t-\tau)}-d_{2}\right)-E y+L_{2}(y-y(t-\tau)), \\
0=E(v y-w)-m,
\end{gathered}
$$

where $L=\left(\begin{array}{ll}L_{1} & L_{2}\end{array}\right)^{T}$ is the feedback gain.

Under the state delayed feedback control, the Jacobian matrix at the positive equilibrium point $Q_{5}$ is

$$
J_{\mathrm{Q}_{5}}^{c}=\left(\begin{array}{cc}
u_{11} e^{-\lambda \tau}+u_{12}+L_{1}\left(1-e^{-\lambda \tau}\right) & u_{2} \\
u_{3} e^{-\lambda \tau} & u_{4}+L_{2}\left(1-e^{-\lambda \tau}\right)
\end{array}\right) .
$$

Then the character equation corresponding to the matrix $J_{\mathrm{Q}_{5}}^{c}$ is

$$
\begin{aligned}
& {\left[\lambda^{2}-\left(u_{12}+u_{4}+L_{1}+L_{2}\right) \lambda+\left(u_{12}+L_{1}\right)\left(L_{2}+u_{4}\right)\right]} \\
& +\left[\left(L_{2}-u_{11}+L_{1}\right) \lambda+\left(u_{11}-L_{1}\right)\left(u_{4}+L_{2}\right)\right. \\
& \left.\quad-L_{2}\left(u_{12}+L_{1}\right)-u_{2} u_{3}\right] e^{-\lambda \tau}-L_{2}\left(u_{11}-L_{1}\right) e^{-2 \lambda \tau}
\end{aligned}
$$

$=0$. 
For the simplicity of computation, the feedback gain term $L_{1}$ is denoted as $u_{11}$. Then, the character equation (56) is written as

$$
\begin{gathered}
{\left[\lambda^{2}-\left(u_{1}+u_{4}+L_{2}\right) \lambda+u_{1}\left(L_{2}+u_{4}\right)\right]} \\
+e^{-\lambda \tau}\left[L_{2} \lambda-L_{2} u_{1}-u_{2} u_{3}\right]=0 .
\end{gathered}
$$

Assume that (57) has a purely imaginary root $\lambda=i \omega$, and substitute it into the equation, then we obtain the following equation:

$$
\begin{aligned}
\omega^{4}+ & \left(u_{1}^{2}+\left(u_{4}+L_{2}\right)^{2}\right) \omega^{2} \\
& +\left(u_{1} u_{4}-u_{2} u_{3}\right)\left(u_{1} u_{4}+u_{2} u_{3}+2 L_{2} u_{1}\right)=0
\end{aligned}
$$

By simple analysis, we can obtain the following result.

Theorem 11. Under the conditions of Theorem 10, for any time delay $\tau>0$, the equilibrium point $Q_{5}$ of the model (54) is locally asymptotically stable if the feedback gain satisfies the following conditions:

$$
\begin{gathered}
L_{1}=u_{11}, \\
u_{1} u_{4}+u_{2} u_{3}+2 L_{2} u_{1}>0 .
\end{gathered}
$$

In fact, the two differential equations of the differentialalgebraic system (54) can be rewritten as

$$
\begin{gathered}
\frac{d x}{d t}=x\left(\frac{x}{A+x}-d_{1}-x(t-\tau)\right)-\frac{x y}{1+h x}+E_{x} x, \\
\frac{d y}{d t}=y\left(\frac{b x(t-\tau)}{1+h x(t-\tau)}-d_{2}\right)+E_{y} y,
\end{gathered}
$$

where $E_{x}=L_{1}(1-x(t-\tau) / x)$ and $E_{y}=L_{2}(1-y(t-\tau) / y)-E$.

Remark 12. From the practical point of view, $E_{x}$ and $E_{y}$ can be regarded as harvesting efforts for the prey and predator species. From the expression above, it is clear that $E_{x}$ and $E_{y}$ are related to the equilibrium, the past, and present population density. Hence, population densities can be kept stable by changing the strength of harvesting efforts; that is, the state delayed feedback control can be completed by adjusting harvesting efforts for two species. Hence, the state feedback controller can be used to eliminate Hopf bifurcation and drive the model system to stabilize at the interior equilibrium point.

\section{Numerical Simulation}

In this section, we firstly assign some parameter values of the model (7) and provide some numerical simulations to illustrate the effectiveness of the results which have been established in the previous sections. For the purpose of simulation experiments, we mainly use the software MATLAB 7.0.

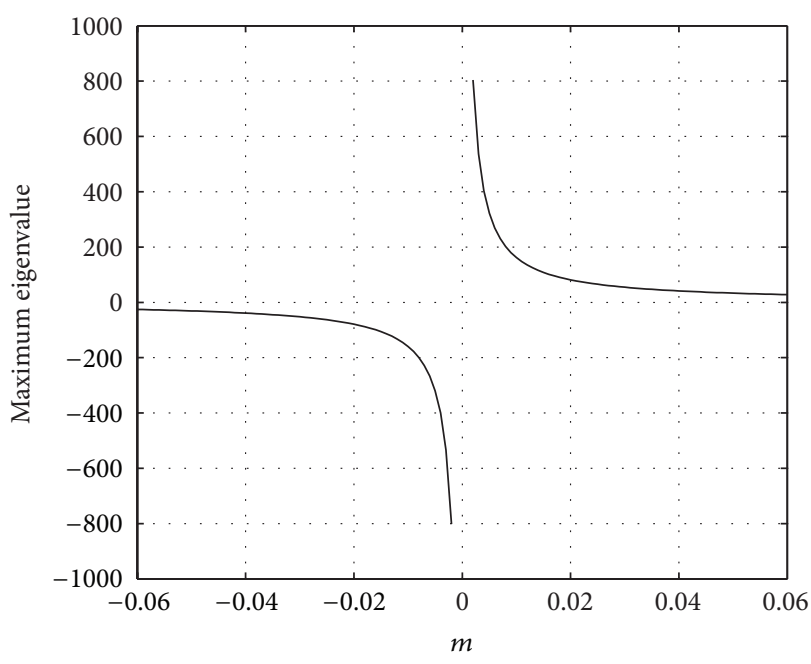

Figure 1: The maximum eigenvalue of the model (61) w.r.t. economic revenue $m$.

The differential-algebraic model (7) with given values takes the following form:

$$
\begin{gathered}
\frac{d x}{d t}=x\left(\frac{x}{A+x}-0.01-x(t-\tau)\right)-\frac{x y}{1+1.25 x}, \\
\frac{d y}{d t}=y\left(\frac{7.5 x(t-\tau)}{1+1.25 x(t-\tau)}-2\right)-E y, \\
0=E(35 y-1)-m .
\end{gathered}
$$

4.1. Existence of Singular Induced Bifurcation. The existence of singularity induced bifurcation of the model (61) is clearly shown in Table 1. From Table 1, it can be seen that as economic revenue $m$ increases through zero, one eigenvalue remains almost constant and the other moves from $C^{-}$to $C^{+}$along the real axis by diverging through $\infty$ for the fixed Allee effect constant. Moreover, for the fixed Allee effect $A=0.02$, we draw a figure to illustrate the movement of maximum eigenvalue with respect to the economic revenue $m$ (see Figure 1). From Figure 1, it can be seen that singularity induced bifurcation for the model (61) occurs at $m=0$, which is corresponding to the theoretical analysis. Hence, the stability of the model (61) at positive equilibrium point changes from stable to unstable. Furthermore, the stronger Allee effect is, the weaker impulsive phenomenon will be; that is, Allee effect has an impact on the dynamical behavior of the proposed model.

4.2. Existence and Control of Hopf Bifurcation. For the fixed parameter $A=0.02$, we draw a critical curve $\tau_{0}^{m}=f(m)$ w.r.t. parameter $m$ (see Figure 2). From Figure 2, it is clear that the domain surrounded by the critical curve $f(m)$ and the two axes is stable for the model (7). Based on the analysis in Section 3.2, the equilibrium point $Q_{5}(0.4055,0.8099,0.0183)$ is stable for the model (7) without time delay. Furthermore, since $p_{2}-p_{4}=-0.7079<0$, there is a positive root $\omega_{m}=$ 0.8781 for (49). The critical value of the delay corresponding 
TABLE 1: Equilibrium points and eigenvalues of the model (7) for different Allee effect constants and economic revenue.

\begin{tabular}{lccc}
\hline Allee effect & Economic profit & Equilibrium point & Eigenvalues \\
\hline \multirow{2}{*}{$A=0.02$} & $m=-0.01$ & $(0.957,0.0284,1.424)$ & $-0.930,-201.571$ \\
& $m=0.01$ & $(0.956,0.0288,1.264)$ & $-0.929,161.025$ \\
\hline \multirow{2}{*}{$A=0.1$} & $m=-0.01$ & $(0.874,0.0285,2.367)$ & $-0.775,-559.217$ \\
& $m=0.01$ & $(0.874,0.0288,1.142)$ & $-0.774,131.598$ \\
\multirow{2}{*}{$A=0.5$} & $m=-0.01$ & $(0.442,0.0265,0.136)$ & $-0.203,-1.684$ \\
& $m=0.01$ & $(0.435,0.0311,0.115)$ & $-0.162,1.420$ \\
\hline
\end{tabular}

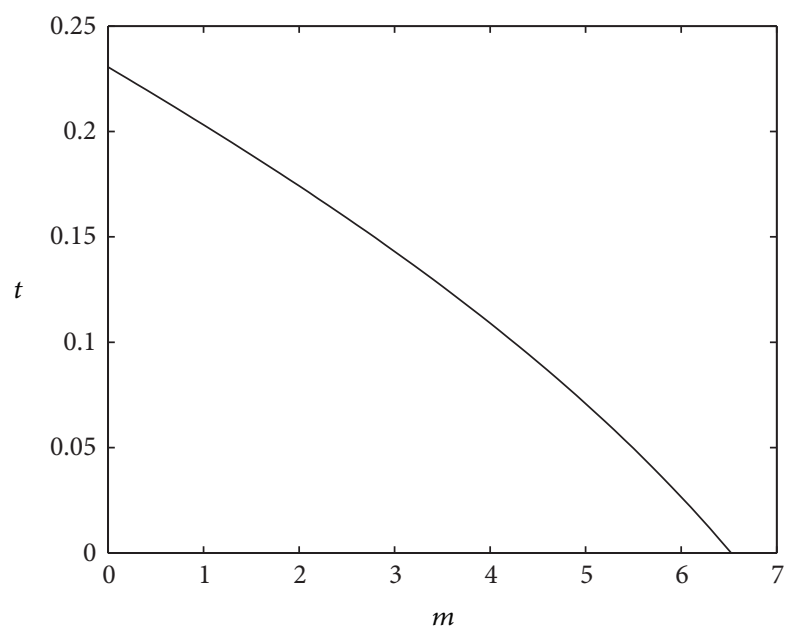

Figure 2: The critical curve of the delay $\tau$ w.r.t. economic revenue $m$.
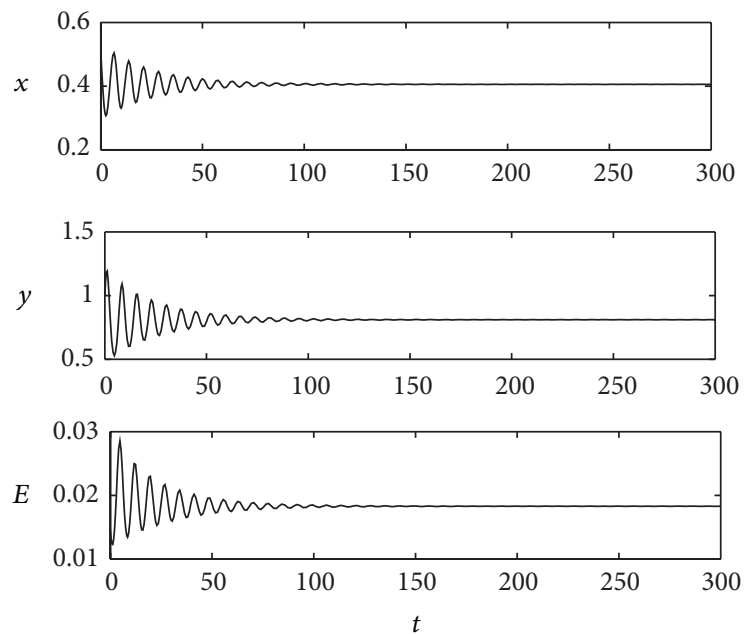

FIGURE 3: When $\tau=0.12$, the equilibrium point $Q_{5}$ is stable.

to $\omega_{m}$ is $\tau_{0}^{m}=0.2172$. The interior equilibrium point $Q_{5}$ remains stable for $\tau<\tau_{0}^{m}$. In Figure 3, a random time delay $\tau=0.12$ is selected in the interval $(0,0.2172)$, which is enough to merit the above mathematical study. When the time delay $\tau$ passes through the critical value $\tau_{0}^{m}$, the equilibrium point $Q_{5}$ loses its stability and Hopf bifurcation occurs. The bifurcating period solution from $Q_{5}$ at $\tau_{0}^{m}$ is

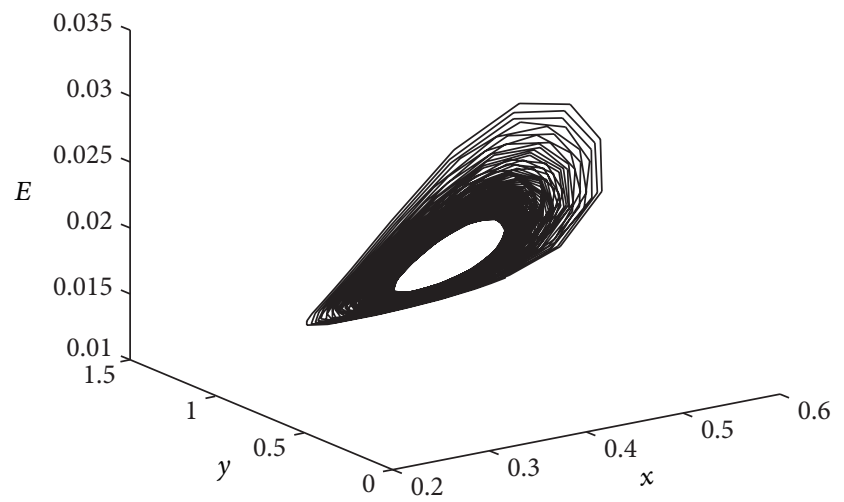

FIGURE 4: When $\tau=0.23$, bifurcation period solutions from the equilibrium point $Q_{5}$ occur.
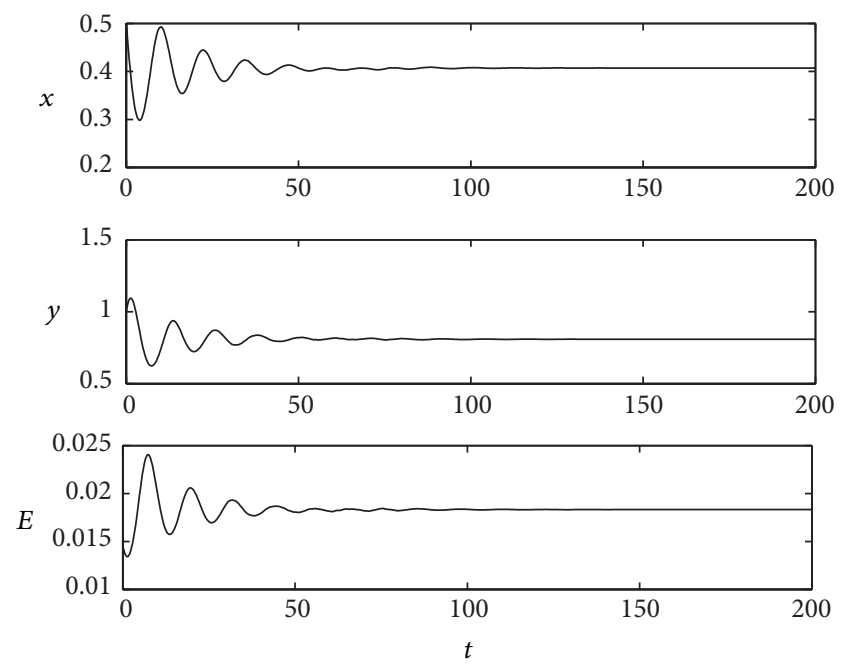

Figure 5: Dynamical responses of the controlled system (54) with the feedback gain $L=\left(\begin{array}{lll}-0.4055 & -7.5\end{array}\right)^{T}$.

depicted in Figure 4. Next, a state feedback controller is applied to the model (7). We choose the feedback gain $L=$ $(-0.4055-7.5)^{T}$, where the gain terms satisfy $L_{1}=u_{11}=$ -0.4055 and $u_{1} u_{4}+u_{2} u_{3}+2 L_{2} u_{1}=1.9754>0$. Then, the model (7) is stable at $Q_{5}$ and the Hopf bifurcation is also eliminated. Figure 5 shows the dynamical responses of the differential-algebraic model (7). 


\section{Conclusions}

Nowadays, much attention has been paid to preserving biological resources with the aim of stemming the damage and ensuring the balance of ecosystems, which inspires the introduction of harvesting in the biological system. In this paper, we analyze the dynamical behavior of a delayed predator-prey model with Allee effect and harvesting by using differential algebraic systems theory. From the analysis of the proposed model, we have obtained some interesting and useful results, which extend the work done in [21]. This paper is mainly divided into two parts.

In the first part, we consider a delayed differentialalgebraic predator-prey model with zero economic revenue. It is observed that transcritical bifurcation and singular induced bifurcation phenomena take place when handling time of predator and economic revenue are regarded as bifurcation parameters, respectively.

As the handling time decreases through the critical point $h_{0}$, the differential-algebraic model undergoes transcritical bifurcation and the stability of $Q_{2}$ is lost. Due to longer handling time $h$ reducing the amount of prey catch per predator per unit of time, prey population density can stay at a positive value and predator population is to be extinct; that is, $Q_{2}$ is stable.

Singular induced bifurcation may cause impulsive phenomenon due to the variation of the economic revenues of harvesting. From a biological point of view, singular induced bifurcation implies rapid expansion of biological population, which may cause ecosystem unbalance and hamper the sustainable development. Hence, it is necessary to investigate the singular induced bifurcation in the presence of a reserve depending on the variation of economic revenue. The analysis of singular induced bifurcation can provide more information of forecasting so that ecological managers can lay down better management strategy. Furthermore, numerical simulation shows that the impulsive phenomenon can be much weaker by increasing Allee effect constant, which implies that Allee effect has an impact on the dynamical behavior of the proposed model. Therefore, ecological managers need to consider the inherent characters of the biological population and some external factors comprehensively.

In general, an individual prey killed does not contribute instantaneously to the growth of predator population. And differential equations with time delay always exhibit much more complicated dynamics than ordinary differential equations. Hence, in the second part of this paper, the effect of time delay on dynamical behavior of the differentialalgebraic model is discussed. It shows that time delay plays an important role in the dynamical behavior of the differential-algebraic model. Hopf bifurcation occurs as time delay increases through a certain threshold, and time delay switches the stability of the proposed model. Furthermore, a state delayed feedback controller is designed to eliminate bifurcational phenomenon and keep population density at steady state. From numerical simulation, it is noted that the stronger Allee effect is not beneficial to the stability of biological species.
It should be noted that almost the existing bioeconomic models (see [13-16]) only investigate the simplest case of a logistic prey growth function. Compared with these works, the introduction of Allee effect makes the work studied in this paper novel.

\section{Conflict of Interests}

The authors do not have a conflict of interests with any commercial identities.

\section{Acknowledgments}

The authors gratefully thank the anonymous authors whose work largely constitutes this paper. This work was supported by the National Science Foundation of China (61273008), Doctor Startup Fund of Liaoning Province (20131026), and Fundamental Research Funds for the Central University (N120405009).

\section{References}

[1] R. M. Etoua and C. Rousseau, "Bifurcation analysis of a generalized Gause model with prey harvesting and a generalized Holling response function of type III," Journal of Differential Equations, vol. 249, no. 9, pp. 2316-2356, 2010.

[2] D. Xiao, W. Li, and M. Han, "Dynamics in a ratio-dependent predator-prey model with predator harvesting," Journal of Mathematical Analysis and Applications, vol. 324, no. 1, pp. 1429, 2006.

[3] N. Bairagi, S. Chaudhuri, and J. Chattopadhyay, "Harvesting as a disease control measure in an eco-epidemiological system-a theoretical study," Mathematical Biosciences, vol. 217, no. 2, pp. 134-144, 2009.

[4] T. Das, R. N. Mukherjee, and K. S. Chaudhuri, "Harvesting of a prey-predator fishery in the presence of toxicity," Applied Mathematical Modelling, vol. 33, no. 5, pp. 2282-2292, 2009.

[5] L. Ji and C. Wu, "Qualitative analysis of a predator-prey model with constant-rate prey harvesting incorporating a constant prey refuge," Nonlinear Analysis: Real World Applications, vol. 11, no. 4, pp. 2285-2295, 2010.

[6] K. Chakraborty, S. Jana, and T. K. Kar, "Global dynamics and bifurcation in a stage structured prey-predator fishery model with harvesting," Applied Mathematics and Computation, vol. 218, no. 18, pp. 9271-9290, 2012.

[7] Z. Xiang, D. Long, and X. Song, "A delayed Lotka-Volterra model with birth pulse and impulsive effect at different moment on the prey," Applied Mathematics and Computation, vol. 219, no. 20, pp. 10263-10270, 2013.

[8] S. Ayasun, C. O. Nwankpa, and H. G. Kwatny, "Computation of singular and singularity induced bifurcation points of differential-algebraic power system model," IEEE Transactions on Circuits and Systems. I, vol. 51, no. 8, pp. 1525-1538, 2004.

[9] R. Riaza, "Singularity-induced bifurcations in lumped circuits," IEEE Transactions on Circuits and Systems. I, vol. 52, no. 7, pp. 1442-1450, 2005.

[10] V. M. Marchenko, O. N. Poddubnaya, and Z. Zaczkiewicz, "On the observability of linear differential-algebraic systems with delays," IEEE Transactions on Automatic Control, vol. 51, no. 8, pp. 1387-1392, 2006. 
[11] L. Chen and K. Aihara, "Global searching ability of chaotic neural networks," IEEE Transactions on Circuits and Systems. I, vol. 46, no. 8, pp. 974-993, 1999.

[12] R. Somogyi and C. A. Sniegoski, "Modeling the complexity of genetic networks: understanding multigenic and pleiotropic regulation," Complexity, vol. 1, no. 6, pp. 45-63, 1995/96.

[13] X. Zhang, Q.-1. Zhang, and Y. Zhang, "Bifurcations of a class of singular biological economic models," Chaos, Solitons \& Fractals, vol. 40, no. 3, pp. 1309-1318, 2009.

[14] W. Liu, C. Fu, and B. Chen, "Hopf bifurcation for a predatorprey biological economic system with Holling type II functional response," Journal of the Franklin Institute, vol. 348, no. 6, pp. 1114-1127, 2011.

[15] K. Chakraborty, M. Chakraborty, and T. K. Kar, "Bifurcation and control of a bioeconomic model of a prey-predator system with a time delay," Nonlinear Analysis: Hybrid Systems, vol. 5, no. 4, pp. 613-625, 2011.

[16] C. Liu, Q. Zhang, X. Zhang, and X. Duan, "Dynamical behavior in a stage-structured differential-algebraic prey-predator model with discrete time delay and harvesting," Journal of Computational and Applied Mathematics, vol. 231, no. 2, pp. 612-625, 2009.

[17] W. C. Allee, Animal Aggretions: A Study in General Sociology, University of Chicago Press, Chicago, Ill, USA, 1931.

[18] H. S. Gordon, "Economic theory of a common property resource: the fishery," Journal of Political Economy, vol. 63, pp. 116-124, 1954.

[19] J. Guckenheimer and P. Holmes, Nonlinear Oscillations, Dynamical Systems, and Bifurcations of Vector Fields, vol. 42, Springer, New York, NY, USA, 1983.

[20] V. Venkatasubramanian, H. Schättler, and J. Zaborszky, "Local bifurcations and feasibility regions in differential-algebraic systems," IEEE Transactions on Automatic Control, vol. 40, no. 12, pp. 1992-2013, 1995.

[21] J. Zu and M. Mimura, "The impact of Allee effect on a predatorprey system with Holling type II functional response," Applied Mathematics and Computation, vol. 217, no. 7, pp. 3542-3556, 2010. 


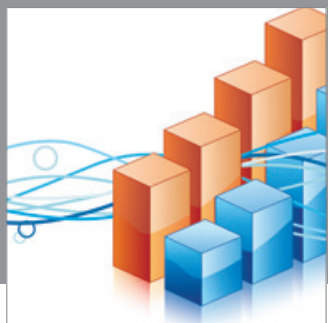

Advances in

Operations Research

mansans

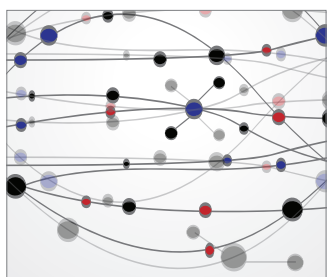

The Scientific World Journal
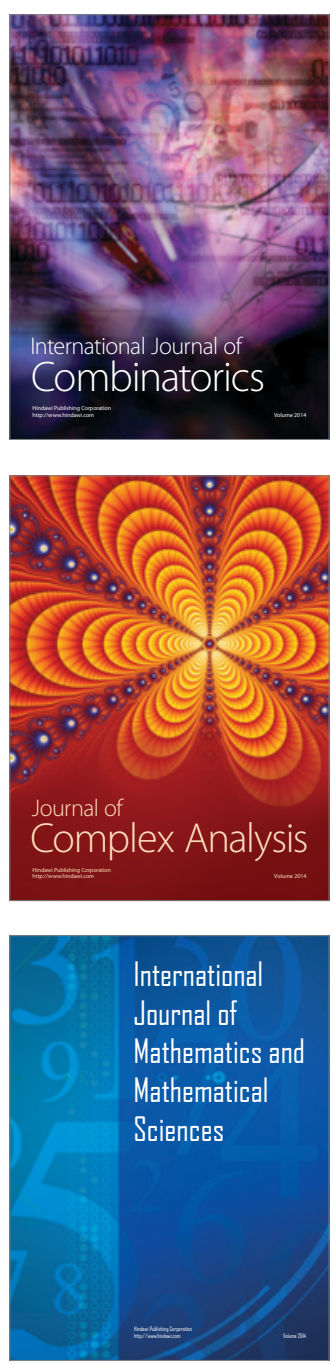
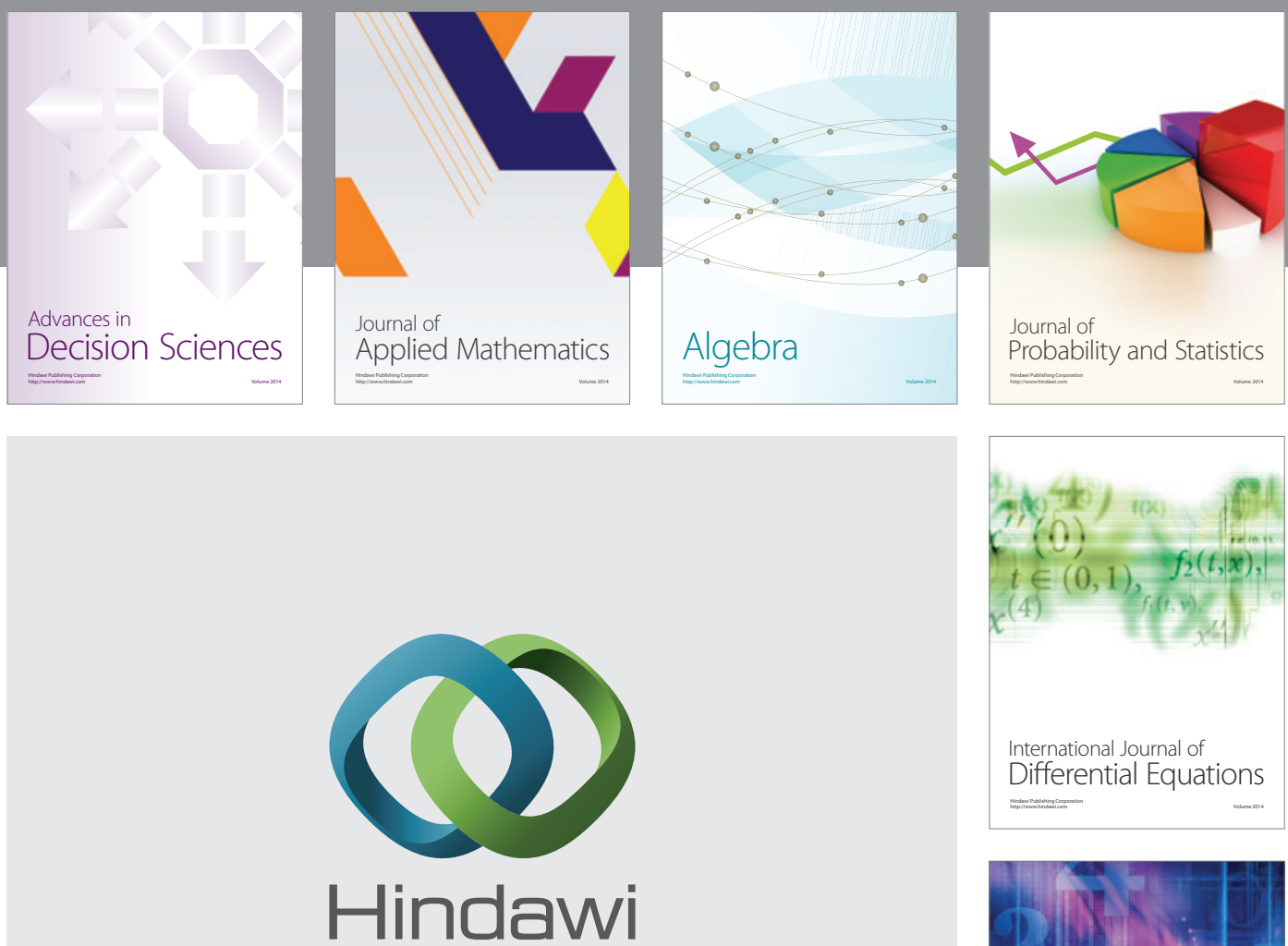

Submit your manuscripts at http://www.hindawi.com
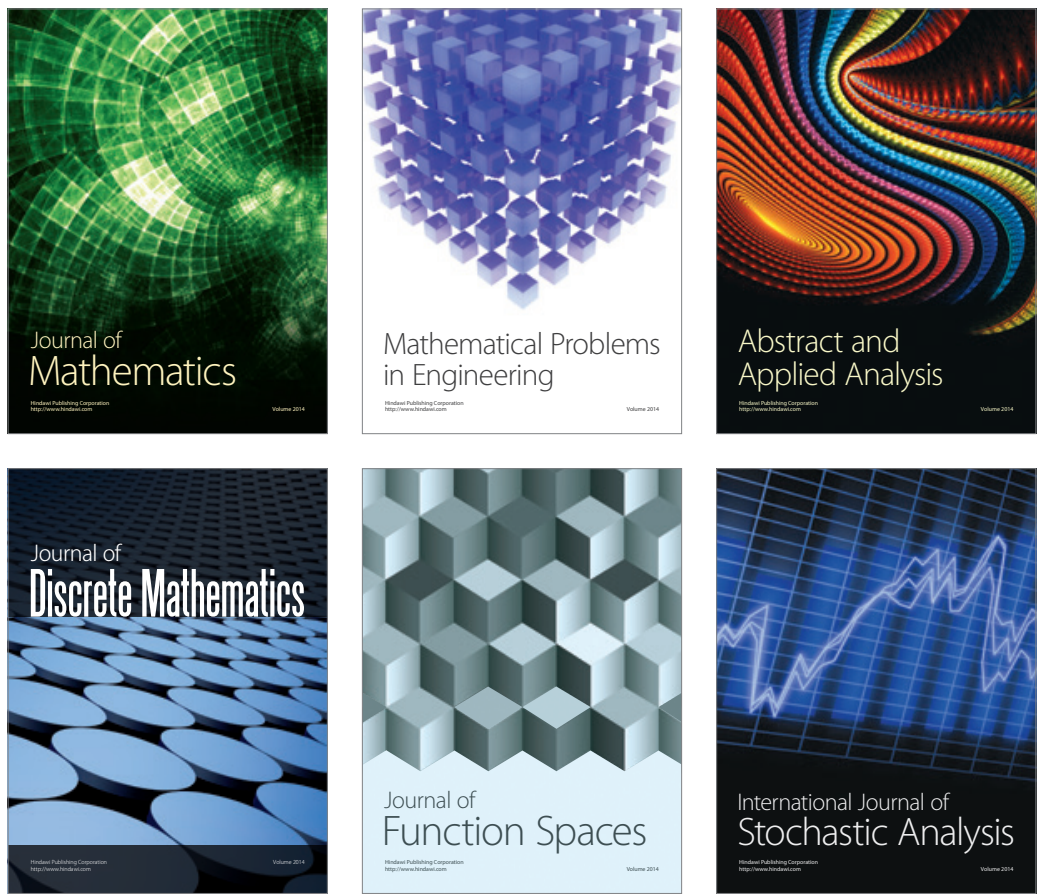

Journal of

Function Spaces

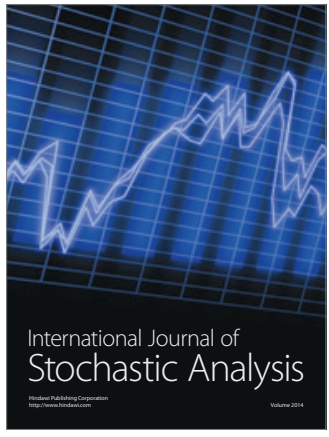

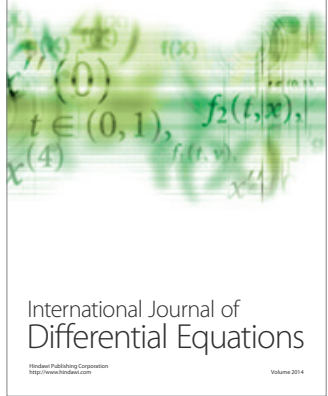
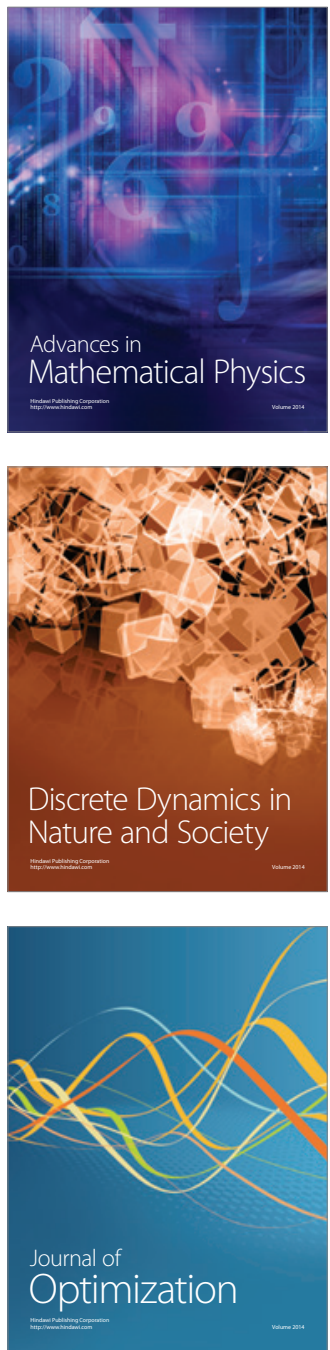